J. Lake Sci. (湖泊科学), 2015, 27(4):629-636

DOI $10.18307 / 2015.0410$

(c) 2015 by Journal of Lake Sciences

\title{
三峡澎溪河高阳平湖高水位时碱性磷酸酶活性及其动力学特征”
}

\author{
张 萍 ${ }^{1}$, 冯 婧 ${ }^{1}$, 李 哲 ${ }^{2,3,4 * *}$, 郭劲松 ${ }^{1,2,4}$, 肖 艳 ${ }^{2,4}$, 刘 静 ${ }^{1}$ \\ ( 1 : 重庆大学城市建设与环境工程学院, 重庆 400045) \\ ( 2 : 中国科学院重庆绿色智能技术研究院,重庆 400030) \\ (3:中国长江三峡集团, 北京 100030$)$ \\ (4: 中国科学院水库水环境重点实验室,重庆 400030)
}

\begin{abstract}
摘 要: 三峡水库汛末蓄水后易出现支流回水区磷累积现象,并在冬季末期常出现硅藻水华现象. 为研究汛末蓄水的磷 积累与冬季末期硅藻水华的相互关系, 分析 2013 年 1-3 月三峡澎溪河高阳平湖库湾水体中碱性磷酸酶活性、磷形态的 转化和藻类生长的协同过程. 结果表明, 总碱性磷酸酶活性 (TAPA) 及其最大反应速率 $\left(V_{\max }\right)$ 、特异性碱性磷酸酶活性 (PAPA/Chl. a) 及 PAPA 与 TAPA 的比值 (PAPA/TAPA) 随着时间推移总体呈先增加后减小而后再增加减小的双峰趋势, 分别在 2 月中旬和 3 月中旬达到峰值. 根据冬季末期水华暴发程的特点将其分为 I 、II III 、IV 4 个阶段. I 阶段为诱导 期, 水体活性磷主要来自藻类碱性磷酸酶分解的有机磷, 藻类对磷过度摄取. II 阶段为过渡期, 温度低, 水体碱性磷酸酶 活性相对较低. III 阶段为水华时期, 水中碱性磷酸酶主要来源于细菌, 叶绿素 a 浓度达到最大, 溶解态反应性磷浓度达到 最低; IV 阶段为水华末期, 水体叶绿素 a 浓度逐渐下降, 溶解态反应性磷浓度回升, 水中碱性磷酸酶主要来源于细菌.

关键词: 三峡水库;高阳平湖;支流库湾; 冬季末期水华;碱性磷酸酶活性;藻类
\end{abstract}

\section{Alkaline phosphatase activity and its kinetics in Lake Gaoyang, Pengxi River during high water level of the Three Gorges Reservoir}

\author{
ZHANG Ping ${ }^{1}$, FENG Jing ${ }^{1}$, LI Zhe ${ }^{2,3,4}$, GUO Jinsong ${ }^{1,2,4}$, XIAO Yan ${ }^{2,4} \&$ LIU Jing $^{1}$ \\ (1: Faculty of Urban Construction and Environmental Engineering, Chongqing University, Chongqing 400045, P. R. China) \\ (2: Chongqing Institute of Green and Intelligent Technology, Chinese Academy of Sciences, Chongqing 400030, P. R. China) \\ (3: China Three Gorges Corporation, Beijing 100030, P. R. China) \\ (4: Key Laboratory of Reservoir Water Environment for Chinese Academy of Sciences, Chongqing 400030, P. R. China)
}

\begin{abstract}
In the tributary backwater area, the Three Gorges Reservoir, impoundment after flooding season often results in phosphorus accumulation. Meanwhile, algal blooms occur almost every year in late winter. To find their relationship, we studied the activity of alkaline phosphatase (alkaline phosphatase activity of phytoplankton-PAPA and total alkaline phosphatase activityTAPA ) and different phosphorus forms during January and March 2013. Results showed that TAPA, maximum reaction rate for total alkaline phosphatase $V_{\max }$, PAPA/TAPA and PAPA/Chl. a flunctuated greatly, reaching the first and second peak at the middle of February and March respectively. According to the characteristics of the algal blooms, we divided it into four periods I, II, III and IV. The first is induction period, during which SPR was mainly from the decomposition of organic phosphorus by alkaline phosphatase of phytoplankton and meanwhile, algae presented excessive intake of phosphate. The second is transition period, when water temperature and alkaline phosphatase activity was relatively low. The third is bloom stage, during which the alkaline phosphatase was mainly from the germs, chlorophyll-a concentration reached the maximum and SPR the minimum. The last is the ending period of the algal bloom, the alkaline phosphatase was also mainly from the germs, chlorophyll-a concentration decreased and soluble reactive phosphorus concentration increased gradually.
\end{abstract}

Keywords: Three Gorges Reservoir; Lake Gaoyang; tributary backwater area; late winter algal blooms; alkaline phosphatase activity; algae

* 国家自然科学基金项目 $(51179215,51309220)$ 和中国科学院西部行动计划项目 (KZCX2-XB3-14) 联合资助. 2014 08-11 收稿;2014-10-17 收修改稿. 张萍(1989～), 女,博士研究生;E-mail : zhangping890511@163. com.

** 通信作者; E-mail: zheli81@ sina. com. 
磷是水生生态系统的重要生源要素, 为藻类等初级生产者的必需营养物质 ${ }^{[1]}$, 但却常为水体初级生产 者生长的限制因子. 通常, 采用钼锑抗分光光度法所测得的溶解性正磷酸盐被称之为溶解态反应性磷 ( soluble reactive phosphorus, SRP), 其他形态的磷只有转化成 SRP 才能够被藻类直接利用. 在上述转化过程中, 碱性磷酸酶可以催化悬浮态颗粒磷和溶解态有机磷 (dissolved organic phosphorus, DOP) 水解成藻类可直接 利用的生物活性磷, 并在浮游植物吸收利用 DOP 的过程中起重要作用 ${ }^{[2]}$.

水体中碱性磷酸酶是由细菌、浮游动植物及其残体在活性磷缺乏时分泌的一种促进水体有机磷矿化的 混合物. 碱性磷酸酶与 SRP 间为典型的 “抑制一诱导” 关系, 即当水体 SRP 缺乏时, 碱性磷酸酶分泌便会增 多、活性增大, 以便将水体中的 DOP 及悬浮态颗粒磷转化成能够被细菌和藻类直接利用的 SRP; 当水体 SRP 充足时, 碱性磷酸酶的分泌及其活性则受到抑制. 其活性高低可反映营养物质转化和能量代谢等的强弱 ${ }^{[3]}$, Matavulj 等 ${ }^{[4-6]}$ 认为水中碱性磷酸酶是正磷酸盐来源的补偿载体, 提出将碱性磷酸酶活性 (APA) 作为评价水 质的新的生化参数. 此外, 碱性磷酸酶还与维持一定的细胞磷及 N/P 比有关 ${ }^{[7]}$. Huang 等 $^{[8]}$ 研究海湾中藻利 用 DOP 与 APA 间的关系, 结果表明碱性磷酸酶在藻利用 DOP 的过程中起着重要的作用.

近年, 碱性磷酸酶与磷的地球化学循环间的关系广受关注. 然而, 研究大多都集中在海洋 ${ }^{[9-10]}$ 、浅水湖 泊 ${ }^{[11-12]}$ 以及土壤 ${ }^{[13]}$ 中碱性磷酸酶分布、性质及活性研究. 三峡水库支流中碱性磷酸酶的相关报道却不多 见 ${ }^{[14]}$, 有关三峡水库中碱性磷酸酶与磷形态转化间的关系更是鲜见报道. 本文选择三峡中段澎溪河高阳平 湖为研究区域, 研究冬季高阳平湖碱性磷酸酶与不同磷形态转化的关系, 以期说明冬末水华暴发与冬季蓄 水导致的磷积累有直接联系.

\section{1 材料与方法}

\section{1 样品采集}

前期研究表明,冬季高水位期间澎溪河高阳平湖冬季末水华主要发生在每年的 $2-3$ 月, 3 月下旬消失. 该期间水华现象主要与同期易出现持续约 2 周的冬旱 ${ }^{[15]}$ 以及连晴回暖的气象条件有关.

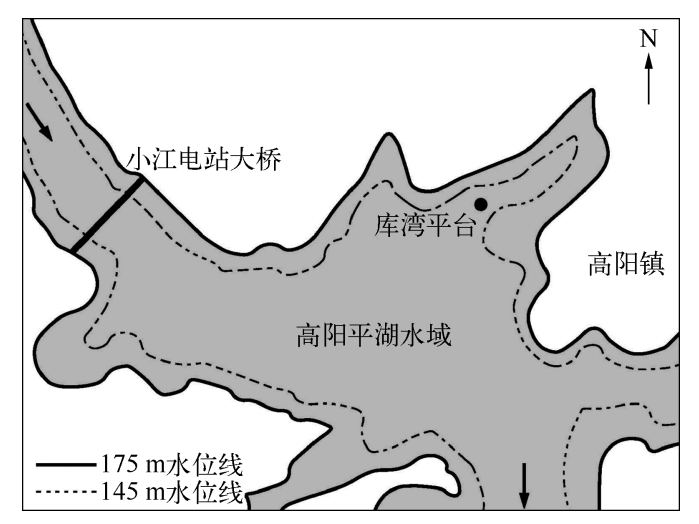

图 1 高阳平湖库湾野外试验平台位置

Fig. 1 The field experiment platform in Lake Gaoyang

为研究水华暴发前至水华结束期间水体中碱性 磷酸酶活性及藻-磷关系, 实验时间设为 2013 年 13 月, 涵盖整个冬季末期水华易发季节并延续至冬 季末期水华现象结束, 地点选在水体流动相对较弱 且较易发生水华的高阳平湖李家坝库湾野外试验平 台 $\left(31.104^{\circ} \mathrm{N}, 108.674^{\circ} \mathrm{E}\right.$, 图 1).

Trimbee 等 ${ }^{[16-17]}$ 认为藻类群落结构 (多样性和演 替速率) 对外界扰动响应显著的时段为 $5 \sim 15 \mathrm{~d}$; Reynolds ${ }^{[18]}$ 研究表明淡水水体中藻类世代周期一般 为 $3 \sim 4 \mathrm{~d}$, 两到三倍世代周期 $(6 \sim 16 \mathrm{~d})$ 可反映藻类 群落演替特征. 因此, 选择采样频次为 $1-2$ 月每月 2 次, 3 月水华暴发, 每周 1 次. 采样时间为每天 $9: 30-16: 30$. 为了把握磷库垂向空间特征, 采用分 层采样, 水样分别采自 $0.5 、 5 、 10 、 20 、 30 、 35 \mathrm{~m}$ 水深 处 (三峡坝前水位为 $163.76 \sim 172.42 \mathrm{~m}$, 此时高阳平湖水深约为 $35 \mathrm{~m}$ ). 采样时将一部分水样放人预先经高 压灭菌的玻璃瓶中用于酶的分析.

\section{2 测定指标及方法}

1.2.1 常规指标测定方法 水温 ( T) 用 YSI63 型 $\mathrm{pH}$ 计现场测得; 水质测定指标有 SRP、溶解性总磷酸盐 (DTP)、DOP、总磷 (TP) 和叶绿素 a ( Chl. a). TP 浓度的测定水样为原水样, 其余指标的测定水样均经 0.45 $\mu \mathrm{m}$ 滤膜抽滤处理. 所有指标的测定均参照《水和废水监测分析方法 (第四版) 》进行 ${ }^{[19]}$.

1.2 .2 水体碱性磷酸酶活性 (TAPA) 和藻类碱性磷酸酶活性 (PAPA) 的测定 碱性磷酸酶能催化水解对硝基 苯磷酸二钠 ( p-NPP) 产生黄色稳定的对硝基苯酚, 通过比色法测定对硝基苯酚的产生速率以确定碱性磷酸酶 
活性 $(\mathrm{APA})^{[20-21]}$. 实验测定方法在李学礼 等 $^{[20-21]}$ 的方法上改进而得: $6 \mathrm{ml}$ 水样加人 $1 \mathrm{ml}$ 浓度为 $0.1 \mathrm{~mol} / \mathrm{L}$ 的 Tris 缓冲液, $2 \mathrm{ml}$ 浓度为 $1 \mathrm{mmol} / \mathrm{L}$ 的 $\mathrm{p}-\mathrm{NPP}$,于 $\mathrm{pH}=8.4$ 、温度为 $30^{\circ} \mathrm{C}$ 条件下反应 $6 \mathrm{~h}$ 后用紫外可见分光光度 计在 $405 \mathrm{~nm}$ 波长下测定. 空白实验采用灭菌蒸馏水代替水样, 其他处理相同. 碱性磷酸酶活性的计算公式为:

$$
A P A=\frac{C}{t \cdot V}
$$

式中, $A P A$ 为碱性磷酸酶活性 $(\mu \mathrm{mol} /(\mathrm{L} \cdot \mathrm{h})), C$ 为水解反应后产生对硝苯酚的量 $(\mu \mathrm{mol}), V$ 为水样的体积 ( $\mathrm{L}), t$ 为反应时间 $(6 \mathrm{~h})$.

忽略天然水体中浮游动物体内的碱性磷酸酶, 故理论上采用 $3.0 \mu \mathrm{m}$ 滤膜 (Whatman 公司) 过滤可将藻 类与细菌进行分离. 过滤后滤液中的碱性磷酸酶活性为细菌碱性磷酸酶和溶解性碱性磷酸酶活性之和 $(\mathrm{BAPA}+\mathrm{DAPA})$, 上述值同未过滤水样所测得的总碱性磷酸酶活性 $(\mathrm{TAPA})$ 之差可视为水中藻类生物体的 碱性磷酸酶活性, 即 PAPA $=$ TAPA - (BAPA + DAPA $)$.

1.2 .3 碱性磷酸酶动力学参数测定设置 $0.5 、 1.0 、 1.5 、 2.0 、 2.5$ 和 $3.0 \mathrm{mmol} / \mathrm{L}$ 共 6 个不同的底物 $(\mathrm{p}-\mathrm{NPP})$ 浓度, 分别测定碱性磷酸酶的活性 (APA), 进而通过 Michaelis-Menten 方程求得碱性磷酸酶动力学参数:

$$
V=\frac{V_{\mathrm{max}} \cdot C_{\mathrm{s}}}{K_{\mathrm{m}}+C_{\mathrm{s}}}
$$

式中, $V$ 为碱性磷酸酶的反应速率 $(\mu \mathrm{mol} /(\mathrm{L} \cdot \mathrm{h})), K_{\mathrm{m}}$ 为米氏常数 $(\mu \mathrm{mol} / \mathrm{L}), C_{\mathrm{s}}$ 为底物浓度 $(\mu \mathrm{mol} / \mathrm{L})$.

应用 Leneweaver-Burk 作图法 ${ }^{[22]}$, 将 Michaelis-Menten 方程两边分别取倒数得公式(3), 通过最小二乘法 求得回归直线斜率 $K_{\mathrm{m}} / V_{\max }$ 和截距 $1 / V_{\text {max }}$, 最终求解动力学参数 $K_{\mathrm{m}}$ 和 $V_{\max }$, 水华暴发期间碱性磷酸酶动力学 参数拟合结果如图 2 所示.

$$
\frac{1}{V}=\frac{K_{\mathrm{m}}}{K_{\max }} \cdot \frac{1}{C_{\mathrm{s}}}+\frac{1}{V_{\max }}
$$
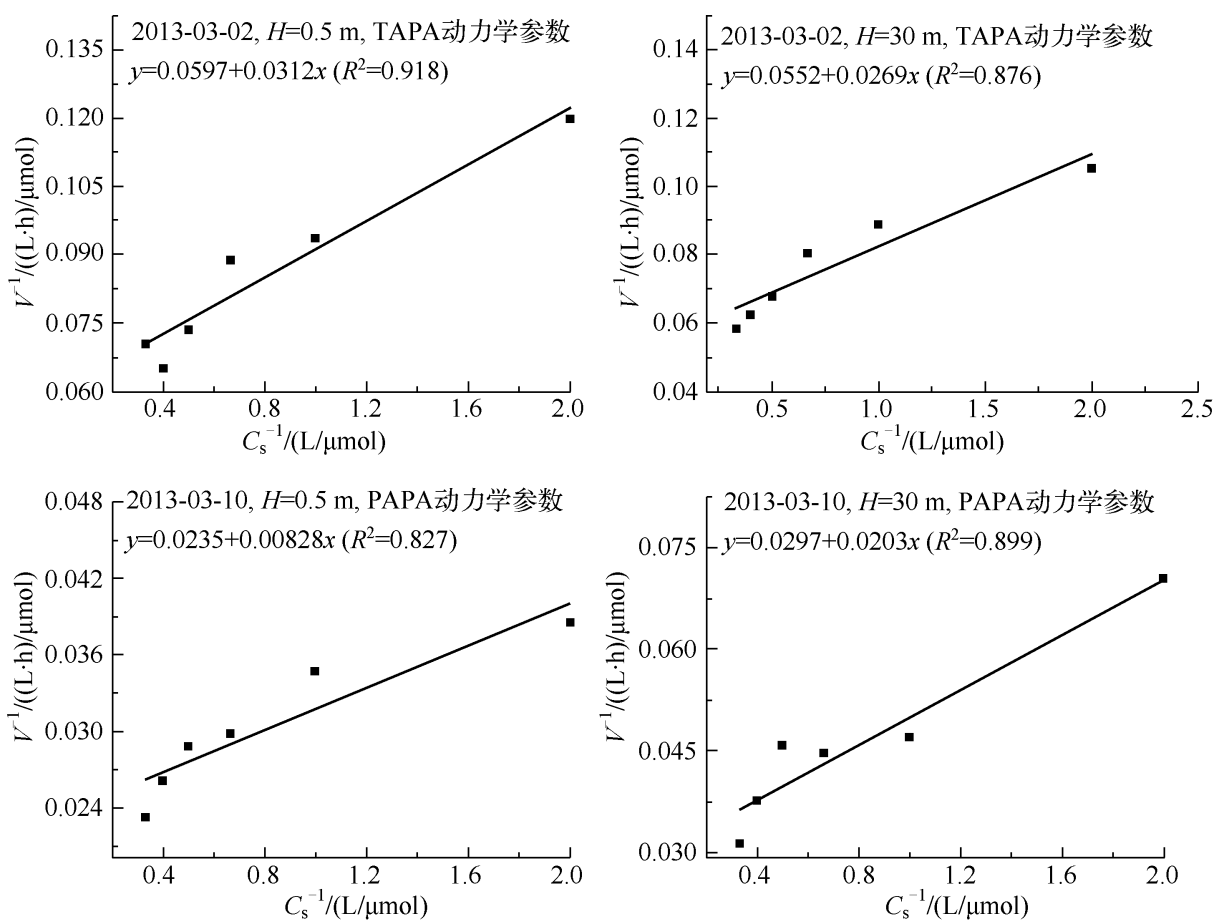

图 2 水华期高阳平湖碱性磷酸酶动力学参数拟合

Fig. 2 Kinetic linear regression of TAPA and PAPA during the algal bloom in Lake Gaoyang 


\section{2 结果与讨论}

2.1 结果

2.1.1 水体总碱性磷酸酶活性及其动力学参数的时空变化 研究期间高阳平湖 TAPA 和 $V_{\max }$ 的均值分别为 $11.20 \pm 6.53$ 和 $30.45 \pm 19.55 \mu \mathrm{mol} /(\mathrm{L} \cdot \mathrm{h})$. TAPA 和 $V_{\text {max }}$ 随时间变化差异极显著 $(P<0.01)$, 研究期间, $\mathrm{TAPA}$ 变化范围为 $4.75 \sim 28.62 \mu \mathrm{mol} /(\mathrm{L} \cdot \mathrm{h})$, 变异系数为 $58.29 \%$, 呈现出先小幅增加, 随后下降而后再次 增加的趋势, 并在 3 月中旬出现最大值. 总碱性磷酸酶 $V_{\text {max }}$ 的变化范围为 $12.83 \sim 87.35 \mu \mathrm{mol} /(\mathrm{L} \cdot \mathrm{h})$, 变异系 数为 $64.20 \%$, 其变化趋势基本与 TAPA 一致, 于 2 月中旬出现小幅度增加并在 3 月中旬出现最大值 (图 3).
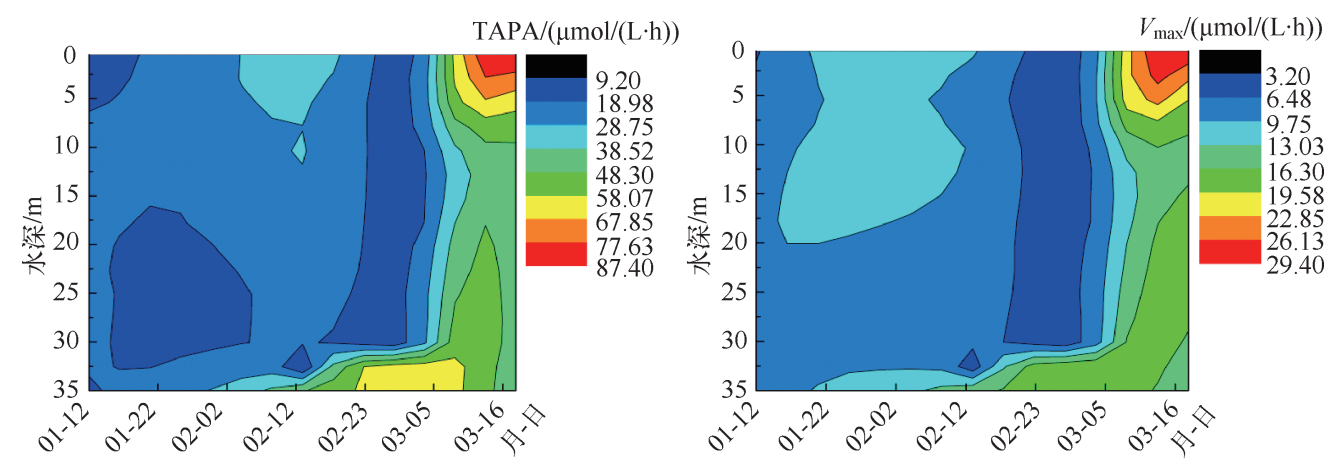

图 3 高阳平湖 TAPA 和 $V_{\text {max }}$ 的垂向变化

Fig. 3 Vertical variations of TAPA and $V_{\max }$ in Lake Gaoyang

研究期间不同水层的 TAPA 及其 $V_{\max }$ 差异不显著 $(P>0.05)$, 水体混合均匀, 分层现象不明显, 仅在水华 暴发期和水华后期 ( 3 月上旬至 3 月中旬) 同时出现了较明显的垂向分层, 差异较显著 $(P<0.05)$, 这与该时 期水温出现的弱分层以及水华暴发时水体中 Chl. a 浓度出现的分层相吻合.

2.1 .2 水体中藻类碱性磷酸酶活性及其动力学参数的时空变化 采用藻类碱性磷酸酶活性与叶绿素 a 所代 表的生物量的比值 (PAPA/Chl. a) 作为特异性活性来表征单位生物量所表现的活性,从实质上反映酶的内在 催化效力. 研究期间高阳平湖水体中特异性碱性磷酸酶活性 (PAPA/Chl. a)、藻类碱性磷酸酶 $V_{\text {max }}$ 和 PAPA/ TAPA 的均值分别为: $1.05 \pm 1.06 \mathrm{~mol} /(\mathrm{mg} \cdot \mathrm{h}) 、 14.78 \pm 13.32 \mu \mathrm{mol} /(\mathrm{L} \cdot \mathrm{h})$ 和 $36.91 \% \pm 14.99 \%$. 从时间分 布上来看, PAPA/Chl. a、 $V_{\max }$ 和 PAPA/TAPA 随着时间的变化差异极显著 $(P<0.01)$, 研究期间, PAPA/Chl. a 的变化范围为 $0.093 \sim 3.680 \mathrm{~mol} /(\mathrm{mg} \cdot \mathrm{h})$, 变异系数为 $101.15 \%$, 总呈现先增加后减少再增加的变化趋势, 于 1 月下旬和 3 月中旬呈现出两个峰值; 藻类碱性磷酸酶 $V_{\text {max }}$ 的变化范围为 $0.50 \sim 60.87 \mu \mathrm{mol} /(\mathrm{L} \cdot \mathrm{h})$, 变 异系数为 $90.14 \%$, 其变化趋势与 PAPA/Chl. a 变化趋势大体一致, 在 2 月中旬出现小幅上升后下降并在 3 月中旬出现最大值; PAPA/TAPA 的最大值出现在 2 月中旬, 并在 3 月中旬出现一个短暂增长后回落 (图 4).

空间上, 研究期间不同水深的 PAPA/Chl. a、藻类碱性磷酸酶 $V_{\max }$ 和 PAPA/TAPA 差异并不显著 $(P>$ 0.05 ), 水体混合均匀, 分层不明显, 仅藻类碱性磷酸酶 $V_{\max }$ 在水华后期 ( 3 月中旬至下旬) 的表层水体中出现 纵向递减现象, 但不同水层之间差异并不显著, 这与此时水温出现弱分层以及水体 Chl. a 浓度出现的分层 一致.

2.1 .3 不同形态磷浓度、叶绿素 a 和水温的时空变化研究期间, 水体中 TP、SRP、DOP、Chl. a 和水温的均值 分别为: $0.071 \pm 0.012 \mathrm{~g} / \mathrm{m}^{3} 、 0.044 \pm 0.015 \mathrm{~g} / \mathrm{m}^{3} 、 0.011 \pm 0.007 \mathrm{~g} / \mathrm{m}^{3} 、 8.60 \pm 10.85 \mathrm{mg} / \mathrm{L}$ 和 $13.33 \pm$ $1.86^{\circ} \mathrm{C}$, 其时空变化趋势如图 5 所示. 从时间分布上来看, 在整个研究期间, TP、SRP 和 DOP 浓度均呈先减小 后增大的趋势, 其变化范围分别为 $0.049 \sim 0.096 、 0.010 \sim 0.058$ 和 $0 \sim 0.026 \mathrm{~g} / \mathrm{m}^{3}$, 其中 DOP 浓度在 1 月下 旬至 2 月下旬期间降到整个研究期间最低值 $\left(0.0036 \pm 0.0019 \mathrm{~g} / \mathrm{m}^{3}\right)$, SRP 浓度在 3 月中旬降至最低 $\left(0.014 \pm 0.0020 \mathrm{~g} / \mathrm{m}^{3}\right)$, 而后逐渐升高; Chl. a 浓度的变化范围为 $1.15 \sim 47.85 \mathrm{mg} / \mathrm{L}$, 其变化趋势却同 SRP 浓度变化趋势相反, 总体呈先增加后下降的趋势, 在 3 月中旬达峰值 $\left(29.64 \pm 14.27 \mathrm{~g} / \mathrm{m}^{3}\right)$, 而后逐渐下降; 


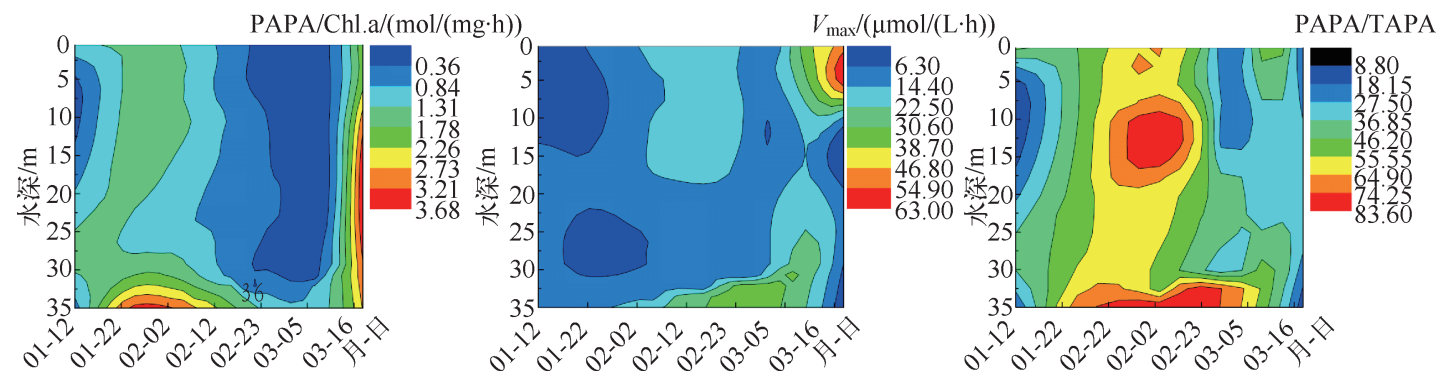

图 4 高阳平湖 PAPA/Chl. a、碱性磷酸酶 $V_{\text {max }}$ 和 PAPA/TAPA 的垂向变化

Fig. 4 Vertical variations of PAPA/Chl. a, $V_{\max }$ and PAPA/TAPA in Lake Gaoyang

水温的变化范围为 $11.2 \sim 18.9^{\circ} \mathrm{C}$, 其变化趋势与 $\mathrm{TP}$ 和 SRP 浓度的变化趋势一致. 从空间分布上来看,在整 个研究期间不同水深的 TP、SRP 和 DOP 浓度差异并不显著 $(P>0.05)$, 水质混合较均匀,水质分层现象总体 并不明显. 随着气温回暖,在研究末期 ( 3 月中旬至下旬) 水温出现弱分层现象, 受温度和光照的影响 Chl. a 浓度也在该时段出现一定的分层,垂向空间差异并不显著 $(P>0.05)$.
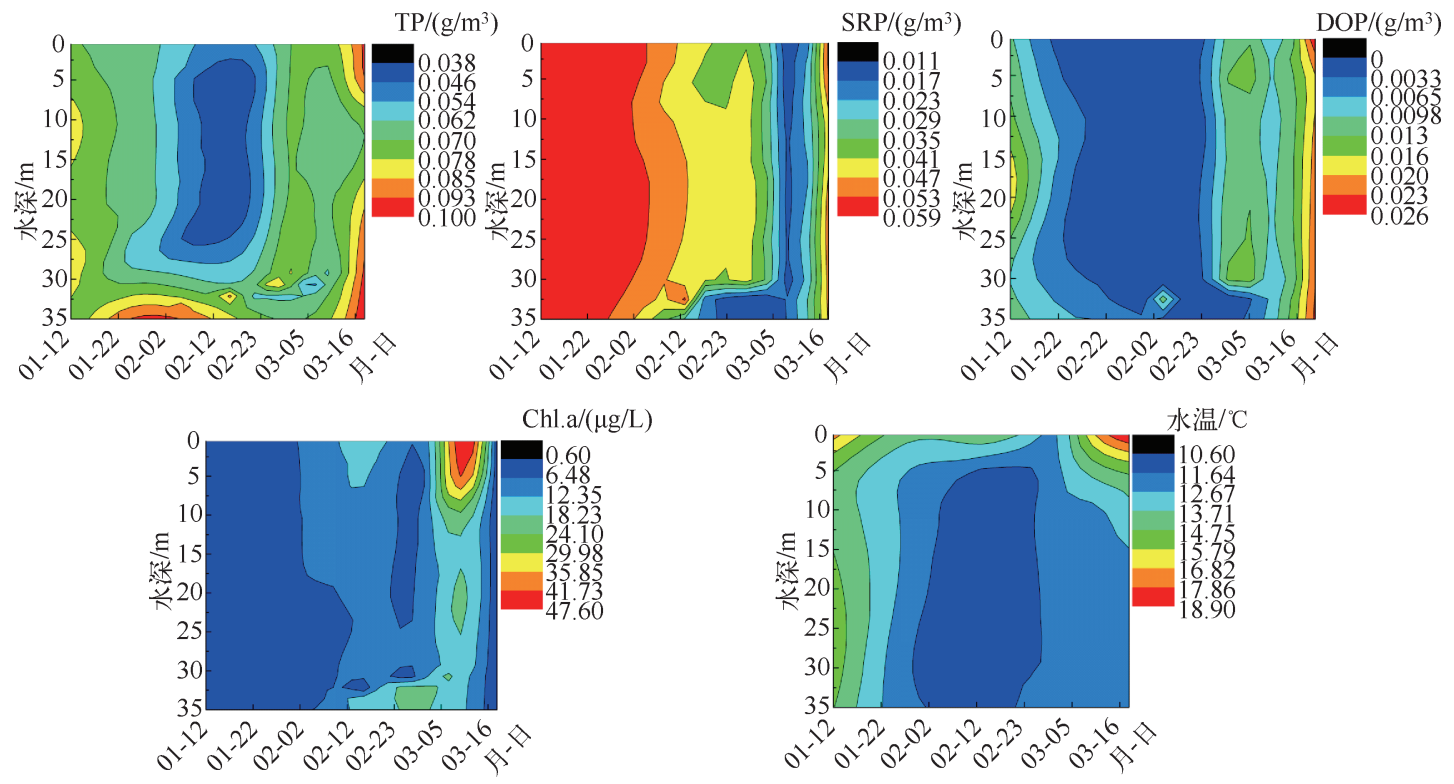

图 5 高阳平湖 TP、SRP、DOP、Chl. a 浓度和水温的垂向变化

Fig. 5 Vertical variations of TP, SRP, DOP, Chl. a concentrations and water temperature in Lake Gaoyang

\section{3 分析与讨论}

TAPA 及其 $V_{\max } 、$ PAPA 及其 $V_{\max }$ 、各种形态磷浓度、Chl. a 浓度等均随时间变化显著,这主要是受温度和 水体活性磷的影响. 温度可以影响酶活性, 低温抑制酶活性, 一定范围内温度升高则会使酶活性增加, 周易 勇等 ${ }^{[23]}$ 研究表明水体碱性磷酸酶活性表达的最佳温度范围为 $25 \sim 40^{\circ} \mathrm{C}$, 温度过高或过低均不利于活性的 表达. SRP 则通过与碱性磷酸酶间的“抑制一诱导” 关系来影响其含量及活性, 从而影响水体中各种形态的磷 浓度; 由于 SRP 是藻类等直接利用的磷营养盐, 因而其与水体 Chl. a 浓度呈现出典型的正相关关系. 它们在 纵向上差异较小, 表明研究期间水体总体混合较均匀; 然而水华后期 (3 月中旬至下旬), 水体呈现较明显的 垂向分布, 可能是由水温的弱分层及光照变化引起 Chl. $\mathrm{a}$ 呈现出分层的原因引起, 这也与图 2 中 $0.5 \mathrm{~m}$ 处的 
藻类碱性磷酸酶内在催化活性高于 $30 \mathrm{~m}$ 处的研究结果相符. 水华暴发时 (约 3 月中旬), 水体表层 Chl. a 浓 度陡增, 且整个水体呈现出较明显的分层现象, 由于能够被藻类利用的磷主要是 SRP, 因此, 水华暴发时, 水 体 SRP 含量降到了最低(图 5).

磷素通常是水体的限制性营养盐, 能够被藻类和细菌直接利用的活性磷 SRP 仅占总磷的 $5 \%$ 左右 ${ }^{[24]}$. $V_{\text {max }}$ 和 $K_{\mathrm{m}}$ 是酶的两个动力学参数, $V_{\text {max }}$ 表征酶的内在催化速率, 而 $K_{\mathrm{m}}$ 表征对底物的亲和力, 其值越小则表明 对底物的亲和力越强 ${ }^{[21]}, K_{\mathrm{m}} / V_{\text {max }}$ 则可以表征 DOP 的周转时间, 其值越小, DOP 的周转时间越短 ${ }^{[25]}$. 表 1 显 示, 实验中 TAPA 及其动力学参数 $V_{\text {max }}$ 与 SRP 浓度呈显著负相关, 这和碱性磷酸酶与 SRP 间的“抑制一诱导” 机制相符, 当水体 SRP 浓度不能满足细菌和藻类生长需要时, 细菌或藻类便会分泌碱性磷酸酶以分解溶解 性有机磷和悬浮态有机磷从而使水体中 SRP 得到补偿 ${ }^{[2-27]}$, 水体碱性磷酸酶活性增大, 催化反应速率加快; 当 SRP 充足时, 其活性便会受到抑制, 藻类、细菌等分泌碱性磷酸酶的能力亦受到抑制. 此外, TAPA 与 Chl. a 浓度呈显著正相关 (表 1), 这与 Jones 的研究相符 ${ }^{[28]}$, 表明总碱性磷酸酶含量可以在一定程度上表征水体生 物量. TAPA 与水温、DOP、TP 浓度均呈正相关, 总碱性磷酸酶 $V_{\text {max }}$ 与各环境变量间的相互关系与 TAPA一 致. TAPA 及其 $V_{\text {max }}$ 与环境因子间呈现的相关关系均为温度、SRP、磷素等综合影响的结果.

表 $1 \mathrm{TAPA}$ 及其动力学参数 $V_{\text {max }}$ 与环境因子的相关性分析

Tab. 1 Correlation analysis among TAPA, $V_{\max }$ and the environmental factors

\begin{tabular}{cccccccc}
\hline 环境因子 & TP & SRP & Chl. a & 水温 & TAPA & 总碱性磷酸酶 $V_{\max }$ & DOP \\
\hline TP & 1 & & & & & & \\
SRP & - & 1 & & & & & \\
Chl. a & - & $-0.861^{* *}$ & 1 & & & & \\
水温 & $0.431^{*}$ & - & - & 1 & & & \\
TAPA & $0.444^{*}$ & $-0.354^{*}$ & $0.445^{*}$ & $0.482^{* *}$ & 1 & & \\
总碱性磷酸酶 $V_{\max }$ & $0.380^{*}$ & $-0.449^{* *}$ & $0.467^{* *}$ & $0.458^{* *}$ & $0.965^{* *}$ & 1 & 1 \\
DOP & $0.702^{* *}$ & - & - & $0.351^{*}$ & $0.367^{*}$ & $0.398^{*}$ & 1 \\
\hline
\end{tabular}

*表示在 0.05 水平 (双侧) 上显著相关, **表示在 0.01 水平(双侧)上显著相关, 一表示无显著相关性.

根据碱性磷酸酶及环境因子的变化规律, 可初步将 2013 年高阳平湖高水位水华期划分成 4 个阶段. I 阶段 ( 1 月中旬至 2 月中旬) 为水华暴发前期, 当 SRP 浓度降到 $0.057 \mathrm{~g} / \mathrm{m}^{3}$ 时, 水体中 SRP 浓度已不能满足 藻类对磷浓度的吸收要求, 藻类被诱导产生大量 AP, 特异性碱性磷酸酶活性相对较高, 期间水体中 AP 主要 是藻类碱性磷酸酶, 约占 $50 \%$. 由此可知, 研究期间高阳平湖藻类对 SRP 浓度降低的反应较细菌敏感, 这与 Fitzgerald 等 ${ }^{[29]}$ 研究结果一致. II 阶段 ( 2 月中旬至 3 月上旬), 水体中 SRP 浓度已不能满足藻类和细菌的需 求, 但此时水温太低不利于碱性磷酸酶活性的表达 ${ }^{[30]}$, 水体中 TAPA 和 PAPA/Chl. a 及其相关动力学参数值 均较低. III 阶段 ( 3 月上旬至中旬), 光热条件适合藻类生长, 冬季末暴发水华, 水体中藻类、浮游动物和细菌 等对 SRP 消耗增加, SRP 浓度持续降低, 当降至 $0.041 \mathrm{~g} / \mathrm{m}^{3}$ 时, SRP 已不能满足细菌生长需要, 细菌被刺激 而大量分泌碱性磷酸酶, TAPA 逐渐增大, PAPA/Chl. a 较小, 在 3 月中旬 SRP 降至最低值、Chl. a 浓度达到最 大值, 水体中的碱性磷酸酶主要由细菌产生. IV 阶段 (3 月中旬至下旬), 冬季末水华结束, 藻类逐渐死亡, TAPA 达到最大以分解藻类合成的有机物质, SRP 浓度逐渐回升, 水中碱性磷酸酶主要来自于细菌. 文中藻 类对水体缺磷的响应阈值相比高光等 ${ }^{[31]}$ 研究太湖得到的藻类碱性磷酸酶分泌阈值大, 这也体现了三峡水库 水体的独特性. 水华期各阶段内碱性磷酸酶及环境因子均值变化如图 6 所示.

水体中碱性磷酸酶诱导-抑制机制复杂, 在 I 阶段特异性磷酸酶活性升高, 水体中碱性磷酸酶以 PAPA 为主, 而在水华暴发的 III 阶段,特异性碱性磷酸酶活性却相对较低. 水华和特异性碱性磷酸酶合成时间出现 不匹配现象, 可能是因为 $\mathrm{PAPA}$ 受 $\mathrm{PO}_{4}^{3-}$ 浓度的显著影响, 而细菌碱性磷酸酶由于 $K_{\mathrm{m}}$ 值较大, 受 $\mathrm{PO}_{4}^{3-}$ 抑制不 明显 ${ }^{[30-31]}$, 因此藻类对 $\mathrm{PO}_{4}^{3-}$ 的响应较细菌敏感. I 阶段水体 $\mathrm{PO}_{4}^{3-}$ 浓度降低, 由于 $\mathrm{PAPA}$ 对其响应敏感, 使得 藻类碱性磷酸酶大量分泌且活性较高; 水华暴发的 III 阶段由于水体 $\mathrm{PO}_{4}^{3-}$ 的大量消耗, 水体活性磷降至细菌 碱性磷酸酶作用的阈值, 致使对 $\mathrm{PO}_{4}^{3-}$ 浓度变化不太敏感的细菌亦明显感到活性磷的缺乏而分泌大量的碱性 
磷酸酶,此时在细菌一藻类之间形成一个短程循环, 同时由于细菌分泌的大量碱性磷酸酶的抑制作用, 使得 藻类分泌碱性磷酸酶的能力减弱, 因而水华暴发期间水体碱性磷酸酶主要来自于细菌.
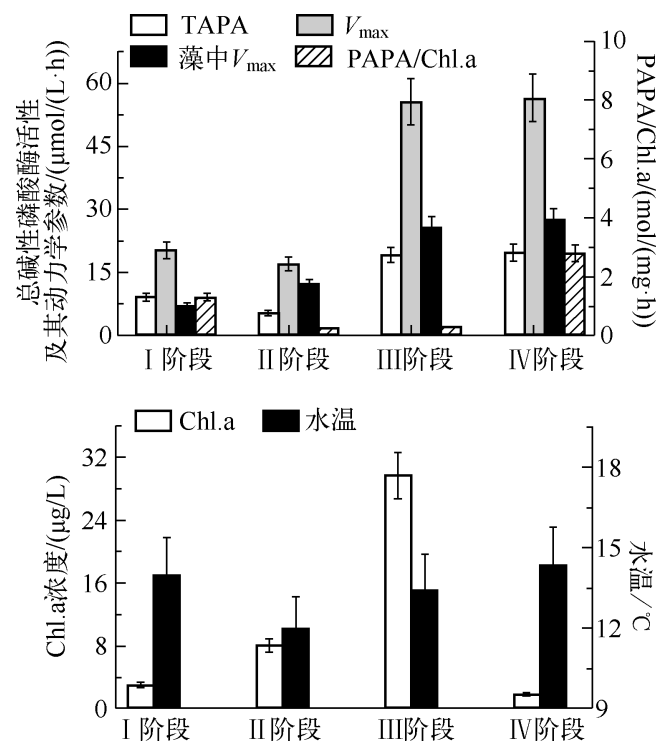

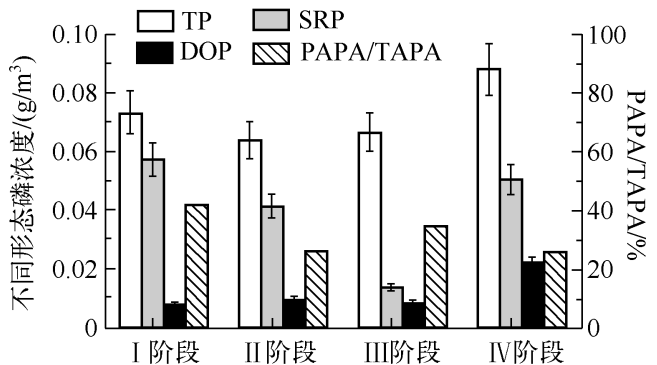

图 6 高阳平湖高水位水华期不同阶段碱 性磷酸酶及环境因子均值变化

Fig. 6 Changes of average TAPA and different environmental factors during algal blooms in Lake Gaoyang

\section{4 结论}

1) 2013 年三峡高水位高阳平湖冬末水华过程中, PAPA/TAPA 及其动力学参数 $V_{\text {max }}$ 水体 TAPA 及其 $V_{\text {max }}$ 均呈现出先增加后减小再增加的趋势, 约 2 月中旬和 3 月中旬出现峰值; PAPA/TAPA 先增加后减小, 水 华暴发前期达最大值,表明水华暴发前水体中活性磷主要来自藻类碱性磷酸酶; 水体 Chl. a 浓度呈先增加后 减小的趋势, 于 3 月中旬达到最大, SRP 浓度变化趋势则与 Chl. a 浓度相反; 影响藻类和细菌碱性磷酸酶合 成的 SRP 浓度分别为 0.057 和 $0.041 \mathrm{~g} / \mathrm{m}^{3}$, 表明藻类对于磷浓度降低的反应较细菌敏感.

2) 根据碱性磷酸酶活性变化特征, 高阳平湖冬末水华过程可分为 4 个阶段. I 阶段为诱导期,水体活性 磷主要来自藻类碱性磷酸酶分解的有机磷, 藻类对磷过度摄取. II 阶段为过渡期, 温度低, 水体碱性磷酸酶 活性相对较低. III 阶段为水华时期,水中碱性磷酸酶主要来源于细菌, Chl. a 浓度达到最大, SRP 浓度达到最 低. IV 阶段为水华末期, 水体 Chl. a 浓度逐渐下降, SRP 浓度回升, 水中碱性磷酸酶主要来源于细菌.

\section{5 参考文献}

[ 1 ] Wetzel RG. Limnology-Lake and river ecosystems: third edition. New York: Academic Press, 2001 :266-269.

[ 2 ] Chrost RJ, Siuda W, Halemejko GZ. Long term studies on alkaline phosphatase activity (APA) in a lake with fish-aquaculture in relation to lake eutrophication and phosphorus cycle. Archiv fiur Hydrobiologie Supplement, 1984, 70 (1) : 1-32.

[ 3 ] Nannipier P, Bollag JM. Use of enzymes to detoxify pestucide-contaminated soils and waters. Journal of Environmental Quality, 1991, 20(3):510-517.

[ 4 ] Matavulj M, Gajin S, Gantar M et al. Phosphatase activity as an additional parameter of water condition estimate in some lakes of Vojvodina Province. Actaboilingosl, 1984, 21 (1):53-62.

[ 5 ] Matavulj M. The nonspecific phosphomonoester-hydrolases of microorganisms and their significance in phosphorus cycle in aquatic environments[Dissertation]. Serbian: University of Zagreb, 1986.

[ 6 ] Matavulj M, Gajin S, Erbeznik M et al. Phosphatase activity of water as a parameter of the river tisa water monitoring. Tiscia (Szeged) , 1989, $23: 29-36$.

[ 7 ] Waiser MJ, Robarts RD. Microbial nutrient limitation in prairie saline lakes with high sulfate concentration. Limnology and Oceanography, 1995, 40 (3) :566-574.

[ 8 ] Huang BQ, Hong HS. Alkaline phosphatase activity and utilization of dissolved organic phosphorus by algae in subtropical 
coastal waters. Marine Pollution Bulletin, 1999, 39(1-12):205-211.

[ 9 ] Huang B, Ou L, Wang X et al. Alkaline phosphatase activity of phytoplankton in East China Sea coastal waters with frequent harmful algal bloom occurrences. Aquatic Microbial Ecology, 2007, 49(2) : 195.

[10］唐洪杰,杨茹君,张传松等. 几种海洋微藻的碱性磷酸酶性质初步研究. 海洋科学,2006,30(10):61-64.

[11] 徐 爽, 刘存歧, 董梦荟等. 白洋淀水体和沉积物中碱性磷酸酶活性的时空分布及其影响因素. 环境科学学报, $2013, \mathbf{3 3}(12): 3317-3323$.

[12] 张成艳,成小英, 王建军等. 傀儡湖水体中碱性磷酸酶活性的时空变化及其影响因素. 应用与环境生物学报,2013, 19 (3) :489-494.

[13] 姜经梅, 赵 慧, 沈铭能等. 长江口潮滩表层沉积物中碱性磷酸酶活性及其影响因素. 环境科学学报, 2011,31 (10):2233-2239.

[14] 袁轶君,毕永红,朱孔贤等.三峡水库沉积物中碱性磷酸酶的活性. 环境科学与技术, 2014,37(1):60-64.

[15] http://www. cqmb. gov. cn/ecms/qixiangfuwu/skfb/2008-12-29/318. html.

[16] Trimbee AM, Harris G. Use of time-series analysis to demonstrate advection rates of different variables in a small lake. Journal of Plankton Research, 1983, 5(6) :819-833.

[17] Gaedeke A, Sommer U. The influence of the frequency of periodic disturbances on the maintenance of phytoplankton diversity. Oecologia, 1986, 71(1) :25-28.

[18] Reynolds CS. Scales of disturbance and their role in plankton ecology. Hydrobiologia, 1993, 249(1-3):157-171.

[19] 国家环境保护总局《水和废水监测分析方法》编委会. 水和废水监测分析方法: 第 4 版. 北京: 中国环境科学出版 社, 2002:243-285.

[20］李学礼,周晓霞. 生物化学与分子生物学实验技术教程. 上海: 同济大学出版社,2008.

[21] 高 光, 朱广伟, 秦伯强等. 太湖水体中碱性磷酸酶的活性及磷的矿化速率. 中国科学: D 辑: 地球科学, 2005, 35 ( II ) : 157-165.

[22] 戚以政,汪叔雄. 生化反应动力学与反应器:第 2 版. 北京: 化学工业出版社, 1999 .

[23] 周易勇,付永清. 水体磷酸酶: 来源,特征及其生态学意义. 湖泊科学, 1999,11(3):274-282.

[24] Halemejko GZ. The role of phosphatases in phosphorus mineralization during decomposition of lake phytoplankton blooms. Archiv fir Hydrobiologie, 1984, 101(4) :489-502.

[25] Reynolds S, Wolff GA, Williams RG et al. Phosphorus cycling in the North and South Atlantic Ocean subtropical gyres. Nature Geoscience, 2008, 1 (7):439-443.

[26] 孔繁翔,宋立荣. 蓝藻水华形成过程及其环境特征研究. 北京:科学出版社,2011.

[27] Hapman A, Foster I, Lees J et al. Particulate phosphorus transport by sub-surface drainage from agricultural land in the UK. Environmental significance at the catchment and national scale. Science of the Total Environment, 2001, 266(1):95102.

[28 ] Jones JG. Studies on freshwater micro-organisms: phosphatase activity in lakes of differing degrees of eutrophication. The Journal of Ecology, 1972, 60 :777-791.

[29] Fitzgerald GP, Nelson TC. Extractive and enzymatic analyses for limiting or surplus phosphorus in algae. Journal of Phycology, 1975, 11 (S1):32-37.

[30] Healey F, Hendzel L. Fluorometric measurement of alkaline phosphatase activity in algae. Freshwater Biology, 1979,9 ( 5 ) : 429-439.

[31] 高 光,高锡芸,秦伯强等. 太湖水体中碱性磷酸酶的作用阈值. 湖泊科学, 2000,12(4):353-358. 